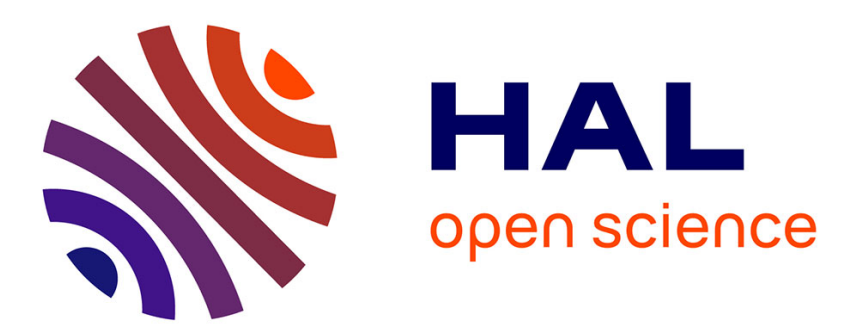

\title{
Single-Trial Classification of Multi-User P300-Based Brain-Computer Interface Using Riemannian Geometry
}

Louis Korczowski, Marco Congedo, Christian Jutten

\section{To cite this version:}

Louis Korczowski, Marco Congedo, Christian Jutten. Single-Trial Classification of Multi-User P300Based Brain-Computer Interface Using Riemannian Geometry. EMBC 2015 - 37th Annual International Conference of the IEEE Engineering in Medicine and Biology Society, Aug 2015, Milan, Italy. 10.1109/EMBC.2015.7318721 . hal-01191913

\section{HAL Id: hal-01191913 https://hal.science/hal-01191913}

Submitted on 3 Sep 2015

HAL is a multi-disciplinary open access archive for the deposit and dissemination of scientific research documents, whether they are published or not. The documents may come from teaching and research institutions in France or abroad, or from public or private research centers.
L'archive ouverte pluridisciplinaire HAL, est destinée au dépôt et à la diffusion de documents scientifiques de niveau recherche, publiés ou non, émanant des établissements d'enseignement et de recherche français ou étrangers, des laboratoires publics ou privés. 


\title{
Single-Trial Classification of Multi-User P300-Based Brain-Computer Interface Using Riemannian Geometry
}

\author{
L. Korczowski, M. Congedo, C. Jutten, Fellow, IEEE
}

\begin{abstract}
The classification of electroencephalographic (EEG) data recorded from multiple users simultaneously is an important challenge in the field of Brain-Computer Interface (BCI). In this paper we compare different approaches for classification of single-trials EventRelated Potential (ERP) on two subjects playing a collaborative BCI game. The minimum distance to mean (MDM) classifier in a Riemannian framework is extended to use the diversity of the inter-subjects spatio-temporal statistics (MDM-hyper) or to merge multiple classifiers (MDM-multi). We show that both these classifiers outperform significantly the mean performance of the two users and analogous classifiers based on the step-wise linear discriminant analysis. More importantly, the MDM-multi outperforms the performance of the best player within the pair.
\end{abstract}

\section{INTRODUCTION}

Computerized systems controlled by EEG cerebral activity have enjoyed a widespread popularity over the past decade [1], but much remains to be done for deploying robust applications to the non-expert public [2]. The introduction of BCI technology is particularly interesting for video gaming, in that the cognitive engagement induced by the gameplay may enhance cognitive processing and could help discriminating relevant cerebral activity [3],[4]. Currently, multiplayer gaming is a steady trend in the gaming industry. Offline studies have shown that multi-user BCI has potential to increase the BCI performance [5] and/or to reduce the length of the single-trial data required for classification, allowing an increased overall effectiveness as compared to single user systems [6]. Recently we have found the classification of single-trial ERPs by Riemannian geometry be very effective [7],[8]. In [9], four different strategies to merge the data collected on several brains simultaneously have been defined. Combining these lines of research, in this paper we study two pipelines for classification of single trial ERP in the context of collaborative video gaming: the minimum distance to mean covariance matrices (MDM) of the whole covariance matrix gathered on the data of two individuals vertically stacked and the MDM obtained on the two individuals separately and successively merged (Figure $1)$. The performance of these classifiers is compared to analogous version of these pipelines obtained using the stepwise linear discriminant analysis (SWLDA).

*This research is partly funded by the European Research Council project CHESS 2012-ERC-AdG-320684.

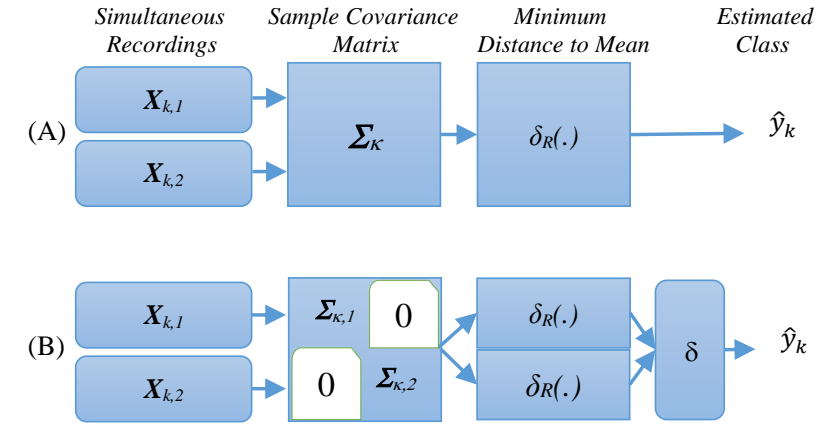

Figure 1 Representation of the two proposed classifiers with a pair of trial $\boldsymbol{X}_{k, 1}$ and $\boldsymbol{X}_{k, 2}$ as input and the estimated class $\hat{y}_{k}$ as output. The "MDM-hyper" (A) described in section II.A consists in computing the extended super covariance matrix (7) and the minimum distance to the center of class defined by (9). The "MDM-multi" (B) consists in two independent MDM classifiers with a voting stage as described in section II.B.

\section{METHODS}

In the context of ERP, the contribution of ERP components that are phase-locked to the visual stimulations is low compared to the background activity and the artifacts (i.e. muscular activity, ocular movement, etc.). Thus, the singletrial detection of an ERP is non-trivial and many approaches attempt to enhance the signal-to-noise ratio by spatial filtering [10] or, more recently, by spatio-temporal filtering [11]. However, the variability across sessions of optimal spatial filters may be non-negligible due to variation of electrode positioning. Although we can assume that the ERP response is more or less constant within the same individual, the variability of the shape, amplitude and latency of ERPs is considerable across subjects, greatly limiting the possibility of transfer learning [7],[8]. In this context, the classification of covariance matrices in the framework of Riemannian geometry, which bypass the estimation of spatial filters, has been shown to be very effective and robust (1rst place at the BCI Challenge NER 2015 and 1rst place at the competition DeMeg2014 - Decoding the Human Brain).

We assume that each trial $\boldsymbol{X}_{k} \in \mathbb{R}^{N \times T}$, where $N$ is the number of electrodes and $T$ the number of sample, is short enough to be considered as a stationary process following a multivariate normal distribution with zero mean. Such a process is defined exhaustively by its covariance matrix, which samples estimation on the $k^{\text {th }}$ trial is

L. Korczowski, M. Congedo, C. Jutten are with the Univ. Grenoble Alpes, GIPSA-lab, F-38000 Grenoble, France (e-mail: louis.korczowski@gipsalab.grenoble-inp.fr) 


$$
\boldsymbol{\Sigma}_{X_{k, m}}=\frac{1}{N-1} \boldsymbol{X}_{k, m} \boldsymbol{X}_{k, m}^{\prime^{\prime}},
$$

with $m \in\{1, \ldots, M\}$ and $M$ the number of subjects. However, such covariance matrix does not hold temporal information at all [8], which is essential to detect phaselocked ERP components. In order to embed temporal information, we estimate the subject's ensemble average ERP response to a target stimulus $\boldsymbol{P}_{m}$ such as

$$
\boldsymbol{P}_{m}=\frac{1}{\left|K^{+}\right|} \sum_{k \in K^{+}} \boldsymbol{X}_{k, m},
$$

where $k$ is the index of the trial, $|K+|$ the number of target trials used to estimate the ensemble average and $K^{+}$the TARGET class. We then build a "super" trial $\widetilde{\boldsymbol{X}}_{k, m}$ by vertical stacking of $\boldsymbol{P}_{m}$ and $\boldsymbol{X}_{k, m}$, such as:

$$
\widetilde{\boldsymbol{X}}_{k, m}=\left[\begin{array}{c}
\boldsymbol{P}_{m} \\
\boldsymbol{X}_{k, m}
\end{array}\right] .
$$

Thus, we can estimate the "super" trial sample covariance matrix (SCM) as

$$
\widetilde{\boldsymbol{\Sigma}}_{k, m}=\left[\begin{array}{cc}
\boldsymbol{\Sigma}_{\boldsymbol{P}_{m}} & \boldsymbol{C}_{\boldsymbol{P}_{m} \boldsymbol{X}_{k, m}} \\
\boldsymbol{C}_{\boldsymbol{X}_{k, m} \boldsymbol{P}_{m}} & \boldsymbol{\Sigma}_{\boldsymbol{X}_{k, m}}
\end{array}\right] .
$$

These covariance matrices are formed by the covariance of $\boldsymbol{P}_{m}$, which plays no role in the classification as it is the same for all trials, the covariance of $\boldsymbol{X}_{k, m}$, which holds the spatial information of the current trial and the cross-covariance between $\boldsymbol{P}_{m}$ and $\boldsymbol{X}_{k, m}$, which contains the most useful information, since its contribution will be proportional to the spatio-temporal coincidence between the current trial and the estimated stereotypical response; in the context of ERP classification with two classes (TARGET $\mathrm{K}^{+}$and NONTARGET K'), each super covariance $\widetilde{\boldsymbol{\Sigma}}_{k, m}$ can be classified by finding the minimal distance from two centers of mass, $\widetilde{\Sigma}_{K^{+}}$and $\Sigma_{K^{-}}$, computed on the training set of TARGET and NON-TARGET trials respectively (see section II.C).

In the case of simultaneous recording on $M$ individuals, we assume that the $M$ simultaneous trials are not independent. Indeed, the EEG of different individuals may be synchronized

1. Exogenously, by the common visual stimulation induced by the game, namely the P300 ERP plus other ERPs due to the same visual and auditory environment [12]

2. Endogenously, by the social interaction induced by the collaboration of the subjects on the task.

To the best of our knowledge, so far none of this spontaneous synchronization has been proven to facilitate the P300 detection. As a first approach, we here compare the classification of ERP with extended super covariance matrices including both intra-subject and inter-subject statistics (MDM-hyper), or by merging the distances obtained on two users independently (MDM-multi), as described here below.

\section{A. MDM-hyper}

The rationale of this classifier is the consideration of the $M$ multiple simultaneous trials as the output of a "hyperbrain". Accordingly, the cross-statistics are embedded by concatenating the $M$ super trials simultaneously such as

$$
\widetilde{\boldsymbol{X}}_{k}=\left[\begin{array}{c}
\boldsymbol{P}_{1} \\
\boldsymbol{X}_{k, 1} \\
\boldsymbol{P}_{2} \\
\boldsymbol{X}_{k, 2}
\end{array}\right] .
$$

The related extended super covariance matrix is then

$$
\widetilde{\Sigma}_{k}=\left[\begin{array}{cccc}
\Sigma_{P_{1}} & C_{P_{1} X_{k, 1}} & C_{P_{1} P_{2}} & C_{P_{1} X_{k, 2}} \\
C_{X_{k, 1} P_{1}} & \Sigma_{X_{k, 1}} & C_{X_{k, 1} P_{2}} & C_{X_{k, 1} X_{k, 2}} \\
C_{P_{2} P_{1}} & C_{P_{2} X_{k, 1}} & \Sigma_{P_{2}} & C_{P_{2} X_{k, 2}} \\
C_{X_{k, 2} P_{1}} & C_{X_{k, 2} X_{k, 1}} & C_{X_{k, 2} P_{2}} & \Sigma_{X_{k, 2}}
\end{array}\right]_{\text {(7) }}
$$

A description of all the terms (partitions) in matrix (7) can be found in [8].

\section{B. MDM-multi}

This Riemannian classifier merges the score of the two singleuser classifiers by computing the sum of the two squared distances to their respective centers of mass defined in (8). Therefore, only intra-subject statistics are used for classification (see, Figure 1).

\section{Compute the center of class and distance}

From the training set, we can compute the center of mass of the covariance matrices mentioned above for each class,

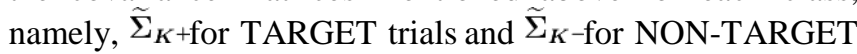
trials by the iterative approach using the log-determinant alpha-divergence function proposed in [13]. Thus, a trial $k$ of an unknown class $y_{k}$ can be classified by finding the minimum distance to the center of mass of each class. To do so, we can use $\delta_{R}$, the Riemannian distance (an affine invariant distance [14]) such as:

$$
d_{C}=\delta_{R}\left(\widetilde{\Sigma}_{C}, \widetilde{\Sigma}_{k}\right) \quad, C \in\left\{K^{+}, K^{-}\right\} .
$$

Finally, the estimated class for each trial is:

$$
\hat{y}=\underset{C}{\operatorname{argmin}}\left(\delta_{R}\left(\widetilde{\Sigma}_{C}, \widetilde{\Sigma}_{k}\right)\right), C \in\left\{K^{+}, K^{-}\right\}
$$

\section{A Collaborative Multi-user BCI}

We designed a collaborative two-user BCI video game based on the video game Brain Invaders [15], inspired to the wellknown vintage video game Space Invaders. The Brain Invaders is based on the oddball paradigm and exploits the detection of a visual P300 evoked potential mush like a P300 speller [1]. Each session of the collaborative Brain Invaders game consisted of nine different levels. At the beginning of the level a grid of $6 \times 6$ aliens was shown, of which one was the TARGET $\left(\mathrm{K}^{+}\right)$(target alien) and the remaining 35 were NON-TARGET $\left(\mathrm{K}^{-}\right)$aliens. The aliens were flashing in random group of six aliens in such a way that after a repetition of 12 flashes each alien had flashed exactly twice. The mean inter-stimulus interval was drawn randomly from an exponential distribution with mean equal to $120 \mathrm{~ms}$, minimum 
of $50 \mathrm{~ms}$ and maximum of $1000 \mathrm{~ms}$. The ERPs of the two users was classified online according to a multi-user version of [7] implemented in Python within the open source software OpenViBE [16]. After each repetition of flashes, the game destroyed the most probable target according to classification output. Once the target alien was destroyed, the players won a number of points inversely proportionally to the number of repetitions needed to destroy the target and the game proceeded to the next level. The players were instructed to maximize their score by completing all the levels using the minimum number of repetitions.

\section{E. Procedures}

EEG signals were recorded synchronously from 64 active electrodes (32 per user) using four USBamp amplifiers [g.Tec, Graz, Austria] with a sampling rate of 512 samples per seconds. The raw EEG were extracted by the OpenViBE 0.12 software [16]. The ground electrodes were placed on $\mathrm{Fz}$ and the reference on the right earlobe. To minimize the triggering jitter, the ERP triggers were recorded in an analog channel synchronized with the EEG data flow by the amplifier itself. The sessions were short (less than 5min) and were separated by a small break during which the participants were invited to rest while being awake. Whereas the experiment involved true online classification, in this paper, we consider only the offline performance in a single-trial training-test set paradigm.

\section{F. Preprocessing (offline)}

From the total 64 EEG channels, 16 channels per user were selected $(\mathrm{N}=16)$, corresponding to typical electrode locations for ERP study [8]: Fp1-Fp2-F5-AFz-F6-T7-Cz-T8-P7-P3-Pz$\mathrm{P} 4-\mathrm{P} 8-\mathrm{O} 1-\mathrm{Oz}-\mathrm{O} 2$ channels. The EEG signals were filtered by a fourth order forward-backward Butterworth band pass filter [1-20] $\mathrm{Hz}$ and down-sampled at $128 \mathrm{~Hz}$. Then, the signals were segmented into epochs of $1 \mathrm{~s}(\mathrm{~T}=128)$ starting at the time instant of the flashes.

\section{G. State-of-the-art methods}

As comparison to the MDM-hyper and MDM-multi Riemannian classifiers, we employ analogous extensions of the stepwise LDA classifier [17] such as:

1) SWLDA-hyper: The epochs were $2 N x T$ and both activities were classified as a "hyperbrain".

2) SWLDA-multi: The NxT trials of each subject were classified independently and the performances were merged by the average of the distances from the hyperplan.

\section{RESULTS}

\section{A. Subjects}

17 pairs of subjects (mean age $=23.1+/-4.2$ ) played to the collaborative version of Brain Invaders during four successive sessions. The sample consisted 34 subjects, 22 males and 12 females. They were the best performing participants of a single-user pilot-study comprising 71 subjects. The pairs were randomly arranged.

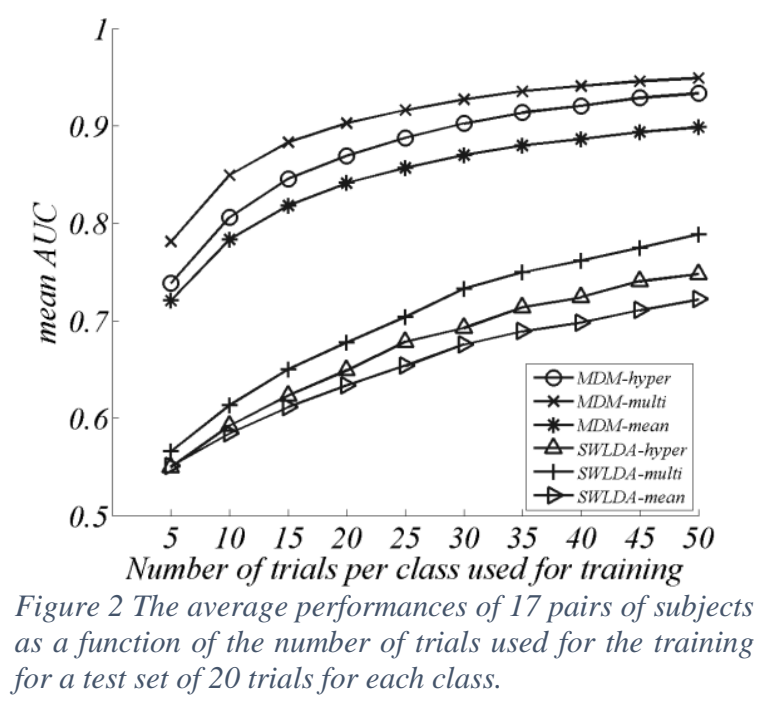

\section{B. Classification performance}

Each recording of four sessions was used to randomly generate 100 unique training-test sets. The Area Under the Curve (AUC) was employed as an index of performance for each classifier. We used the average AUC of the 100 trainingtest sets to estimate the classification scores and we defined the following performance measures:

- $\Pi_{1}, \Pi_{2}$ the AUC of each subject independently related to the classification performances of the $\operatorname{SCM}(5)$

- $\quad \Pi_{\max }=\max \left(\Pi_{1}, \Pi_{2}\right)$, the maximum AUC obtained by either player 1 or 2 of the pair.

- $\Pi_{\text {mean }}=\left(\Pi_{1}+\Pi_{2}\right) / 2$, the average AUC of the pair.

- $\quad \Pi_{\text {hyper }}$ the performance of the MDM-hyper classifier with both intra- and inter-subject statistics, i.e., SCM (7)

- $\quad \Pi_{\text {multi }}$ the AUC of the MDM-multi classifier using only intra-subject statistics of each player and a vote. We use paired Student t-tests for assessing the significance of AUC comparisons. Figure 2 shows the global performance of individual classifiers, $\Pi_{\text {mean }}(\mathrm{MDM}) / \Pi_{\text {mean }}(\mathrm{SWLDA})$ and collaborative classifiers with inter statistics or without $\Pi_{\text {hyper }}$ (MDM) / $\Pi_{\text {hyper }}$ (SWLDA) $\Pi_{\text {multi }}(M D M) / \Pi_{\text {multi }}(S W L D A)$ as a function of the number of trials used for the training set. Even though each online repetition consisted of two target flashes and 10 non-target flashes, we used equitable class numerosity by retaining $1 / 5$ of the non-target flashes chosen randomly. The global performance of MDM classifiers is clearly superior to the performance of the SWLDA classifiers $(t(16)=20.9, p<1 e-4)$. This highly significant difference is probably due to the small size of the training sets, since SWLDA is known to perform well with large training sets [8]. The MDM-multi classifier exhibits the best performance, see Table 1, with a significant increase as compared to the mean performance of the two users $(t(16)=10.3, p<1 e-4)$. Although the absolute difference between the best user and the MDMmulti is small $(+0.021)$, it is significant $(t(16)=2.71, p=1.52 e$ - 
2). Furthermore, the variability of the MDM-multi classifiers across the 100 training-test sets and the 17 pairs is smaller as compared to the variability of single-user classifiers MDMmax (Fisher test : $\Pi_{\text {multi }}$ Versus $\Pi_{\max }, F(1699)=1.80, p<1 e-4$ ). Finally, for both MDM and SWLDA, the difference in performance between the multi-user classifier and the best solo classifier $\left(\Pi_{\operatorname{multi}}-\Pi_{\max }\right)$ depends on the homogeneity of the subjects' performance $\left|\Pi_{1}-\Pi_{2}\right|$, i.e. $\left(\Pi_{\operatorname{multi}}-\Pi_{\max }\right)$ and $\mid \Pi_{1}$ $\Pi_{2} \mid$ are inversely correlated $(r(14)=-0.805, p<1 e-4)$. The influence of performance homogeneity on multi-user classifiers will be investigated in further studies.

Table 1 The average performance (mean AUC) of the MDM classifiers are compared with 100 training-test sets consisting of 20 target and 20 non-target trials.

\begin{tabular}{|c|c|c|c|c|c|c|}
\hline Pair & $\boldsymbol{\Pi}_{\text {hyper }}$ & $\boldsymbol{\Pi}_{\text {multi }}$ & $\boldsymbol{\Pi}_{\text {mean }}$ & $\begin{array}{c}\boldsymbol{\Pi}_{\text {multi- }} \\
\boldsymbol{\Pi}_{\text {mean }}\end{array} \boldsymbol{\Pi}_{\text {max }}$ & $\begin{array}{c}\boldsymbol{\Pi}_{\text {mult- }} \\
\boldsymbol{\Pi}_{\text {max }}\end{array}$ \\
\hline G1 & 0.927 & $\mathbf{0 . 9 5 6}$ & 0.884 & 0.072 & 0.936 & 0.019 \\
\hline G2 & 0.881 & $\mathbf{0 . 9 0 2}$ & 0.836 & 0.066 & 0.898 & 0.004 \\
\hline G3 & 0.879 & $\mathbf{0 . 9 2 9}$ & 0.863 & 0.066 & 0.912 & 0.016 \\
\hline G4 & 0.903 & 0.928 & 0.832 & 0.095 & $\mathbf{0 . 9 3 3}$ & -0.005 \\
\hline G5 & 0.900 & $\mathbf{0 . 9 2 7}$ & 0.873 & 0.054 & 0.884 & 0.043 \\
\hline G6 & 0.887 & $\mathbf{0 . 9 0 1}$ & 0.865 & 0.037 & 0.873 & 0.028 \\
\hline G9 & 0.870 & $\mathbf{0 . 9 0 3}$ & 0.833 & 0.070 & 0.842 & 0.061 \\
\hline G8 & 0.878 & $\mathbf{0 . 9 2 0}$ & 0.852 & 0.068 & 0.883 & 0.037 \\
\hline G9 & 0.791 & 0.837 & 0.764 & 0.073 & $\mathbf{0 . 8 6 8}$ & -0.031 \\
\hline G10 & 0.900 & $\mathbf{0 . 9 3 8}$ & 0.866 & 0.072 & 0.916 & 0.023 \\
\hline G11 & 0.890 & $\mathbf{0 . 9 2 7}$ & 0.858 & 0.070 & 0.868 & 0.060 \\
\hline G12 & 0.898 & $\mathbf{0 . 9 4 4}$ & 0.888 & 0.056 & 0.917 & 0.027 \\
\hline G13 & 0.802 & 0.814 & 0.809 & 0.005 & $\mathbf{0 . 8 5 3}$ & -0.040 \\
\hline G14 & 0.932 & $\mathbf{0 . 9 6 3}$ & 0.907 & 0.056 & 0.931 & 0.032 \\
\hline G15 & 0.843 & $\mathbf{0 . 8 9 4}$ & 0.815 & 0.079 & 0.848 & 0.046 \\
\hline G16 & 0.770 & 0.810 & 0.766 & 0.044 & $\mathbf{0 . 8 3 9}$ & -0.029 \\
\hline G17 & 0.822 & $\mathbf{0 . 8 5 6}$ & 0.792 & 0.065 & 0.792 & 0.064 \\
\hline Mean & 0.869 & $\mathbf{0 . 9 0 3}$ & 0.841 & $\mathbf{0 . 0 6 2}$ & 0.882 & $\mathbf{0 . 0 2 1}$ \\
\hline std & 0.047 & 0.046 & 0.041 & 0.019 & 0.039 & 0.032 \\
\hline p-value & & & & $<1 \mathrm{e}-4$ & & 0.016 \\
\hline
\end{tabular}

\section{CONCLUSION}

Merging EEG activity can be an interesting way to improve BCI system if several users interact simultaneously with the same goal. One usable working hypothesis in this case is that the activity of the multiple players is not independent. We have shown that using our methods (MDM-hyper and MDMmulti) the multi-user performance is significantly superior as compared to the mean performance. The MDM-multi is even superior to the best of the two players taken individually, with the improvement being proportional to the homogeneity of the two players' performance and not to the initial performance itself. Therefore, we bring decisive evidence that a whole new class of classifiers should be designed for detection of coincidental EEG signals in hyperscanning settings. However, in this study, the contribution of the endogenous synchronization has not been directly studied. The effect of collaboration and competition has been assessed in hyperscanning study [18], but not yet with BCI systems, except for sole behavioral observation [9]. The study of exogenous and endogenous brain synchronization in multi- users $\mathrm{BCI}$ is a challenging and fascinating field for future research in BCI.

\section{ACKNOWLEDGMENT}

The experimental protocol has been evaluated and approved by the ethical committee of the University of Grenoble CERNI (2014-01-14-34). The protocol took place in the experimental platform with the approval of the regional health agency (ARS Rhônes-Alpes, ordinance $\mathrm{n}^{\circ} 4603^{\text {rd }}$ June 2010). Thanks: Anton Andreev, engineer in charge of the software plateform. Ekaterina Ostaschenko, experimental support.

\section{REFERENCES}

[1] J. R. Wolpaw, N. Birbaumer, D. J. McFarland, G. Pfurtscheller, and T. M. Vaughan, "Brain-computer interfaces for communication and control," Clin Neurophysiol. Off. J. Int. Fed. Clin. Neurophysiol., vol. 113, no. 6, pp. 767-791, Jun. 2002.

[2] A. Nijholt, B. Reuderink, and D. O. Bos, "Turning Shortcomings into Challenges: Brain-Computer Interfaces for Games," in Intelligent Technologies for Interactive Entertainment, A. Nijholt, D. Reidsma, and H. Hondorp, Eds. Springer Berlin Heidelberg, 2009, pp. 153-168.

[3] R. Leeb, F. Lee, C. Keinrath, R. Scherer, H. Bischof, and G. Pfurtscheller, "Braincomputer communication: motivation, aim, and impact of exploring a virtua apartment," IEEE Trans. Neural Syst. Rehabil. Eng. Publ. IEEE Eng. Med. Biol. Soc., vol. 15 , no. 4 , pp. 473-482, Dec. 2007.

[4] S. C. Kleih, F. Nijboer, S. Halder, and A. Kübler, "Motivation modulates the P300 amplitude during brain-computer interface use," Clin. Neurophysiol. Off. J. Int. Fed. Clin. Neurophysiol., vol. 121, no. 7, pp. 1023-1031, Jul. 2010.

[5] Y. Wang and T.-P. Jung, "A Collaborative Brain-Computer Interface for Improving Human Performance," PLOS ONE, vol. 6, no. 5, p. e20422, May 2011

[6] P. Yuan, Y. Wang, W. Wu, H. Xu, X. Gao, and S. Gao, "Study on an online collaborative BCI to accelerate response to visual targets," in 2012 Annual International Conference of the IEEE Engineering in Medicine and Biology Society (EMBC), 2012, pp. 1736-1739.

[7] A. Barachant and M. Congedo, "A Plug\&Play P300 BCI Using Information Geometry," ArXiv14090107 Cs Stat, Aug. 2014

[8] M. Congedo, "EEG Source Analysis," HDR Thesis, Université de Grenoble, 2013.

[9] L. Bonnet, F. Lotte, and A. Lecuyer, "Two Brains, One Game: Design and Evaluation of a Multiuser BCI Video Game Based on Motor Imagery," IEEE Trans. Comput. Intell. AI Games, vol. 5, no. 2, pp. 185-198, 2013.

[10] B. Rivet, A. Souloumiac, V. Attina, and G. Gibert, "xDAWN Algorithm to Enhance Evoked Potentials: Application to Brain \#x2013;Computer Interface," IEEE Trans. Biomed. Eng., vol. 56, no. 8, pp. 2035-2043, Aug. 2009.

[11] K. Yu, K. Shen, S. Shao, W. C. Ng, K. Kwok, and X. Li, "Common spatiotemporal pattern for single-trial detection of event-related potential in rapid serial visual presentation triage," IEEE Trans. Biomed. Eng., vol. 58, no. 9, pp. 2513 2520, Sep. 2011.

[12] U. Hasson, Y. Nir, I. Levy, G. Fuhrmann, and R. Malach, "Intersubject Synchronization of Cortical Activity During Natural Vision," Science, vol. 303, no. 5664, pp. 1634-1640, Mar. 2004.

[13] Z. Chebbi and M. Moakher, "Means of Hermitian positive-definite matrices based on the log-determinant -divergence function," Linear Algebra Its Appl., vol. 436, no. 7, pp. 1872-1889, Apr. 2012.

[14] X. Pennec, P. Fillard, and N. Ayache, "A Riemannian Framework for Tensor Computing," Int. J. Comput. Vis., vol. 66, no. 1, pp. 41-66, Jan. 2006.

[15] M. Congedo, M. Goyat, N. Tarrin, G. Ionescu, L. Varnet, B. Rivet, R. Phlypo, N. Jrad, M. Acquadro, and C. Jutten, "'Brain Invaders": a prototype of an opensource P300- based video game working with the OpenViBE platform," in 5th International Brain-Computer Interface Conference 2011 (BCI 2011), Graz, Austria, 2011, pp. 280-283.

[16] Y. Renard, F. Lotte, G. Gibert, M. Congedo, E. Maby, V. Delannoy, O. Bertrand, and A. Lécuyer, "OpenViBE: An Open-Source Software Platform to Design, Test, and Use Brain-Computer Interfaces in Real and Virtual Environments," Presence Teleoperators Virtual Environ., vol. 19, no. 1, pp. 35-53, Feb. 2010.

[17] D. J. Krusienski, E. W. Sellers, F. Cabestaing, S. Bayoudh, D. J. Mcfarland, T. M. Vaughan, and J. R. Wolpaw, "A comparison of classification techniques for the P300 speller,” J. Neural Eng., vol. 3, no. 4, pp. 299-305, Oct. 2006.

[18] F. De Vico Fallani, V. Nicosia, R. Sinatra, L. Astolfi, F. Cincotti, D. Mattia, C. Wilke, A. Doud, V. Latora, B. He, and F. Babiloni, "Defecting or Not Defecting: How to 'Read' Human Behavior during Cooperative Games by EEG Measurements," PLoS ONE, vol. 5, no. 12, p. e14187, Dec. 2010. 